\title{
A Constellation Scaling Approach to Vector Perturbation for Adaptive Modulation in MU-MIMO
}

\author{
Ang Li, and Christos Masouros, Senior Member, IEEE
}

\begin{abstract}
It is known that vector perturbation (VP) precoding does not apply to the case where users employ different modulations, while existing solutions for this scenario are suboptimal. In this letter, a joint vector perturbation precoding algorithm is proposed for multiuser MIMO (MU-MIMO) downlink system in the adaptive modulation scenario where different users apply different modulation types. Compared with conventional block diagonalized vector perturbation (BD-VP) and user grouping VP where the search dimension of the perturbation vector is reduced, the proposed algorithm keeps the search dimension unchanged by applying a simple transformation to the VP operation. Our analysis and results show that the proposed algorithm provides an applicable VP solution to the adaptive modulation scenario, with optimal VP performance.
\end{abstract}

Index Terms-MIMO, vector perturbation, precoding, adaptive modulation, joint perturbation

\section{INTRODUCTION}

I $\mathrm{N}$ recent MIMO communications, precoding techniques have been widely studied due to the need for power and cost efficient UE devices. These range from capacity achieving dirty paper coding [1] to linear precoding schemes [2]. Vector perturbation (VP) precoding is proposed to improve the performance of channel inversion and can reach near-capacity performance [3]. However, one drawback of VP precoding is that adaptive modulation where different users apply different modulation types cannot be used due to the constant modulo base $\tau=2|\mathrm{C}|_{\max }+\Delta$ which is modulation dependent, where $|\mathrm{C}|_{\max }$ is the absolute value of the constellation symbol with the maximum magnitude and $\Delta$ denotes the minimum Euclidean distance between constellation symbols. On the other hand, when users employ multiple antennas, the most popular precoding technique is block diagonalization (BD) [4]. In [5], BD and VP precoding are combined and proposed as block diagonalized vector perturbation (BD-VP) which enables different users to apply different modulation types. However, due to the direct combination of these two techniques, the complexity of BD-VP is rather high. The authors in [6] therefore propose a low-complexity BD-VP, and furthermore a user grouping vector perturbation (UG-VP) that improves the performance of the BD-VP precoding.

Manuscript received January 26, 2015, accepted March 2, 2015. The associate editor coordinating the review of this letter and approving it for publication was K. Huang.

A. Li and C. Masouros are with the Dept. of Electronic and Electrical Eng., University College London, Torrington Place, London, WC1E 7JE, UK (e-mail: liang19891216@gmail.com, chris.masouros@ieee.org).

This work was supported by the Royal Academy of Engineering, UK and the Engineering and Physical Sciences Research Council (EPSRC) project EP/M014150/1.
Both BD-VP and UG-VP are suboptimal since perturbation is applied for each user or group independently. In order to eliminate the performance loss, in this letter we propose a joint vector perturbation algorithm which reaches a comparable performance with the conventional VP and can be applied to adaptive modulation scenario. By scaling the constellation of the modulation, the modulo base for different modulation types is made the same. Therefore, the search of the optimal perturbation vector of each users can be performed jointly as in conventional VP. Another advantage of the proposed algorithm is that it forms a basis for the VP precoding technique to be applied with users applying different modulation types. As a result, while out of the scope of this paper, other complexity reduction VP algorithms [7]-[9] can be applied on top of this algorithm for complexity benefits.

Notation: $E(\cdot),(\cdot)^{T},(\cdot)^{H},(\cdot)^{-1}$ and $(\cdot)^{\dagger}$ denote expectation, transpose, conjugate transpose, inverse and MoorePenrose inverse respectively. $\|\cdot\|$ denotes the Frobenius Norm, $I_{n}$ is the $n \times n$ identity matrix and $\mathbf{0}$ denotes zero matrix or vector. $\{\mathbf{X}\}_{a: b}$ denotes the matrix formed from the $a$ th column to the $b$ th column of the matrix $\mathbf{X}$. $\mathbf{C} \mathbf{Z}^{k}$ represents $n \times 1$ vectors in the complex set.

\section{SYSTEM MODEL AND VP}

\section{A. MU-MIMO Channel Model}

We consider a multiuser multi-stream MIMO downlink system where the base station communicates with $K$ users. The base station is equipped with $N_{t}$ antennas and user $k$ is equipped with $n_{k} \geq 1$ antennas. The total number of receive antennas is therefore defined as $N_{\mathrm{r}}=\sum_{k=1}^{K} n_{k}$ with $N_{r} \leq N_{t}$. We assume the channel from the base station to each user to be flat fading and modeled by the $n_{k} \times N_{t}$ channel matrix $\mathbf{H}_{k}$, and the channel matrix from the base station to all users can be expressed as $\mathbf{H}=\left[\mathbf{H}_{1}^{T}, \mathbf{H}_{2}^{T}, \ldots, \mathbf{H}_{K}^{T}\right]^{T}$. The received signal at $k$ th user is

$$
\mathbf{y}_{k}=\mathbf{H}_{k} \mathbf{F}_{k} \mathbf{x}_{k}+\mathbf{H}_{k} \sum_{i=1, i \neq k}^{K} \mathbf{F}_{i} \mathbf{x}_{i}+\mathbf{z}_{k}
$$

where $\mathbf{x}_{k}$ is the transmit signal and $\mathbf{z}_{k}$ is the additive complex Gaussian noise vector with zero mean and unit variance. $\mathbf{F}_{k}$ is the precoding matrix for the $k$ th user.

\section{B. $B D$-VP Precoding}

VP Precoding employs a channel inversion to form the precoding matrix and then applies a perturbation on the 
transmitted signals so that the useful signal at the receiver is maximized. In traditional BD-VP [5], the precoder $\mathbf{F}_{k}$ for the $k$ th user can be expressed as $\mathbf{F}_{k}=\mathbf{B}_{k} \mathbf{D}_{k}$, where $\mathbf{B}_{k}$ is the matrix chosen to eliminate multi-user interference, i.e., $\mathbf{H}_{i} \mathbf{B}_{k}=0$, for $i \neq k$. $\mathbf{B}_{k}$ can be obtained from the singular value decomposition (SVD) of $\tilde{\mathbf{H}}_{k}$ as follows:

$$
\tilde{\mathbf{H}}_{k}=\tilde{\mathbf{U}}_{k} \tilde{\Delta}_{k}\left[\tilde{\mathbf{V}}_{k}^{(1)}, \tilde{\mathbf{V}}_{k}^{(0)}\right]^{H}
$$

where $\tilde{\mathbf{H}}_{k}=\left[\mathbf{H}_{1}^{T}, \ldots, \mathbf{H}_{k-1}^{T}, \mathbf{H}_{k+1}^{T}, \ldots, \mathbf{H}_{K}^{T}\right]^{T} . \mathbf{B}_{k}$ is chosen as $\mathbf{B}_{k}=\tilde{\mathbf{V}}_{k}^{(0)}$ because $\tilde{\mathbf{V}}_{k}^{(0)}$ is the orthogonal basis of the null space of $\tilde{\mathbf{H}}_{k}$. After applying $\mathbf{B}_{k}$, user $k$ now has a noninterfering channel $\mathbf{H}_{k}^{e q u}=\mathbf{H}_{k} \mathbf{B}_{k}$. Based on the idea of channel inversion, $\mathbf{D}_{k}$ is chosen as $\mathbf{D}_{k}=\left(\mathbf{H}_{k} \mathbf{B}_{k}\right)^{-1}$. Thus, the perturbation vector for the $k$ th user is given by

$$
\mathbf{l}_{k}^{\text {opt }}=\underset{\mathbf{l}_{k} \in \mathbf{C} \mathbf{Z}^{k}}{\arg \min }\left\|\left(\mathbf{H}_{k} \mathbf{B}_{k}\right)^{-1} \cdot\left(\mathbf{s}_{k}+\tau_{k} \mathbf{l}_{k}\right)\right\|^{2}
$$

For the low-complexity BD-VP in [6], the precoding matrix for user $k$ can be expressed as $\mathbf{F}_{k}=\hat{\mathbf{H}}_{k}$, and $\hat{\mathbf{H}}_{k}=$ $\left\{\mathbf{H}^{\dagger}\right\}_{(k-1) n_{k}+1: k n_{k}}$. The transmit signal of user $k$ is then given by

$$
\mathbf{x}_{k}=\sqrt{\frac{P}{\beta_{k}}} \hat{\mathbf{H}}_{k}\left(\mathbf{s}_{k}+\tau_{k} \mathbf{l}_{k}\right)
$$

where $\beta_{k}=\left\|\hat{\mathbf{H}}_{k} \cdot\left(\mathbf{s}_{k}+\tau_{k} \mathbf{l}_{k}\right)\right\|^{2}$ denotes the transmit power scaling factor so that $E\left(\left\|\mathbf{x}_{k}\right\|^{2}\right)=P$. $\mathbf{l}_{k}$ belongs to $n_{k}$ dimensions perturbation vector and is given by

$$
\mathbf{l}_{k}^{\text {opt }}=\underset{\mathbf{l}_{k} \in \mathbf{C} \mathbf{Z}^{k}}{\arg \min }\left\|\hat{\mathbf{H}}_{k} \cdot\left(\mathbf{s}_{k}+\tau_{k} \mathbf{l}_{k}\right)\right\|^{2}
$$

At the receiver, the signal is first scaled back to eliminate the effect of the transmit scaling factor, and then fed to a modulo operator to remove the perturbation vector [3].

\section{UG-VP Precoding}

For UG-VP, the basic idea is to divide $K$ users into $B$ groups (denoted as $\left\{G_{1}, G_{2}, \ldots, G_{B}\right\}$ ) based on their modulation type, and perform the vector perturbation within each user group. Users with the same modulation type are divided into the same group, therefore $\tau_{k}^{b}=\tau^{b}, k \in G_{b}, b=1, \ldots, B$. For each group, the optimal perturbation vector is given by

$$
\mathbf{l}_{b}^{o p t}=\underset{\mathbf{l}_{b} \in \mathbf{C Z}^{n_{b}}}{\arg \min }\left\|\hat{\mathbf{H}}_{b} \cdot\left(\mathbf{s}_{b}+\tau_{b} \mathbf{l}_{b}\right)\right\|^{2}
$$

where $\mathbf{H}^{\dagger}=\left[\hat{\mathbf{H}}_{1}, \ldots, \hat{\mathbf{H}}_{B}\right], \hat{\mathbf{H}}_{b}$ is a $n_{b} \times N_{t}$ channel matrix from the base station to the user group $G_{b}, \mathbf{s}_{b}=$ $\left[s_{b_{1}}^{T}, s_{b_{2}}^{T}, \ldots, s_{b_{M}}^{T}\right]^{T}$ and $n_{b}=\sum_{k=b_{1}}^{b_{M}} n_{k}, b_{M}$ is the total number of users in the $b$ th group. By reducing the number of individual search for the optimal perturbation vectors, it is shown in [6] that UG-VP outperforms BD-VP.

\section{Proposed Joint Vector Perturbation (JVP)}

In conventional VP algorithm, the perturbation vector $\mathbf{l}^{\text {opt }}$ is searched over $N_{r}$ dimensions to minimize the total transmit power. However, due to the limitation of the constant value of $\tau$, conventional VP cannot be directly applied to scenarios where users employ multiple modulation types. BD-VP performs a $n_{k}$ dimensions search for $K$ times to obtain the perturbation vector, while UG-VP performs a $n_{b}$ dimensions search for $B$ times. Rewrite $\mathbf{H}^{\dagger}=\left[\hat{\mathbf{H}}_{1}, \ldots, \hat{\mathbf{H}}_{K}\right]$, then we have

$$
\begin{aligned}
\left\|\mathbf{H}^{\dagger}(\mathbf{s}+\tau \mathbf{l})\right\|^{2} & =\left\|\sum_{k=1}^{K} \hat{\mathbf{H}}_{k} \cdot\left(\mathbf{s}_{k}+\tau_{k} \mathbf{l}_{k}\right)\right\|^{2} \\
& \leq \sum_{k=1}^{K}\left\|\hat{\mathbf{H}}_{k} \cdot\left(\mathbf{s}_{k}+\tau_{k} \mathbf{l}_{k}\right)\right\|^{2}
\end{aligned}
$$

It is found that with the increase of $K$, the gap between $\left\|\mathbf{H}^{\dagger}(\mathbf{s}+\tau \mathbf{l})\right\|^{2}$ and $\sum_{k=1}^{K}\left\|\hat{\mathbf{H}}_{k} \cdot\left(\mathbf{s}_{k}+\tau_{k} \mathbf{l}_{k}\right)\right\|^{2}$ is larger [6]. Therefore, it is easy to conclude that although UG-VP performs better than BD-VP, they are both sub-optimal compared with conventional VP.

In order to keep the performance advantage of conventional VP, we propose a joint perturbation that retains the search dimension as $N_{r}$. Without loss of generality, we assume there are multiple modulation types applied, denoted as $\mathcal{A}, \mathcal{B}, \mathcal{C}, \ldots, \mathcal{M}$. For modulation type $i$, the total number of symbols applying this kind of modulation is denoted as $N_{i}$ and $\sum_{i=\mathcal{A}}^{\mathcal{M}} N_{i}=N_{r}$. Rewrite $\mathbf{H}^{\dagger}=\left[\mathbf{H}_{\mathcal{A}}, \ldots, \mathbf{H}_{\mathcal{M}}\right], \mathbf{s}+\tau \mathbf{l}=$ $\left[\left(\mathbf{s}_{\mathcal{A}}+\tau_{\mathcal{A}} \mathbf{l}_{\mathcal{A}}\right)^{T}, \ldots,\left(\mathbf{s}_{\mathcal{M}}+\tau_{\mathcal{M}} \mathbf{l}_{\mathcal{M}}\right)^{T}\right]^{T}$, where $\mathbf{s}_{i}$ denotes the symbols from the constellation of modulation type $i$ and $\mathbf{H}_{i}$ is the corresponding channel matrix. Then, we can reformulate the transmit signal norm as

$$
\begin{array}{r}
\left\|\mathbf{H}^{\dagger}(\mathbf{s}+\tau \mathbf{l})\right\|^{2}=\left\|\left[\mathbf{H}_{\mathcal{A}}, \ldots, \mathbf{H}_{\mathcal{M}}\right] \cdot(\mathbf{s}+\tau \mathbf{l})\right\|^{2} \\
=\left\|\sum_{i=\mathcal{A}}^{\mathcal{M}} \frac{\tau_{i}}{\tau_{0}} \cdot \frac{\tau_{0}}{\tau_{i}} \mathbf{H}_{i}\left(\mathbf{s}_{i}+\tau_{i} \mathbf{l}_{i}\right)\right\|^{2}=\left\|\sum_{i=\mathcal{A}}^{\mathcal{M}} \frac{\tau_{i}}{\tau_{0}} \mathbf{H}_{i}\left(\frac{\tau_{0}}{\tau_{i}} \mathbf{s}_{i}+\tau_{0} \mathbf{l}_{i}\right)\right\|^{2}
\end{array}
$$

where $\mathbf{l}_{i}$ is the perturbation vector for modulation type $i$ and $\tau_{0}$ denotes the modulo base to be used. Based on the derivation above, it is worth noting that there is no special restriction for the choice of $\tau_{0}$. By defining $\mathbf{G}=\left[\frac{\tau_{\mathcal{A}}}{\tau_{0}} \mathbf{H}_{\mathcal{A}}, \ldots, \frac{\tau_{\mathcal{M}}}{\tau_{0}} \mathbf{H}_{\mathcal{M}}\right]$ and $\mathbf{t}=\left[\frac{\tau_{0}}{\tau_{\mathcal{A}}} s_{\mathcal{A}}^{T}, \ldots, \frac{\tau_{0}}{\tau_{\mathcal{M}}} s_{\mathcal{M}}^{T}\right]^{T},(8)$ can be transformed into

$$
\left\|\mathbf{H}^{\dagger}(\mathbf{s}+\tau \mathbf{l})\right\|^{2}=\left\|\mathbf{G}\left(\mathbf{t}+\tau_{0} \mathbf{l}\right)\right\|^{2}
$$

With this transformation, the search for the optimal perturbation vector in the adaptive modulation scenario can be applied within $N_{r}$ dimensions so that there will be no performance loss compared with BD-VP and UG-VP. Intuitively, this algorithm functions as follows: by scaling the constellation of the modulation, different modulation types have the same value of $\tau$ so that they can perform the vector perturbation jointly. Fig. 1 depicts the normalized constellation points of 16QAM, QPSK, and scaled QPSK (sQPSK) to show how our algorithm works. According to the definition of $\tau$ [4], the length of the fold lines $\tau_{16 Q A M}=d_{1}^{16 Q A M}+d_{2}^{16 Q A M}$ and $\tau_{Q P S K}=d_{1}^{Q P S K}+d_{2}^{Q P S K}$ denotes the values of the modulo base for 16QAM and QPSK as per Fig. 1, respectively. As the value of modulo base for original QPSK is larger than that of 16QAM, we scale the constellation of QPSK by $\tau_{16 Q A M} \tau_{\text {QPSK }}$ such that $\frac{\tau_{16 Q A M}}{\tau_{S Q P S K}}=\frac{d_{1}^{16 Q A M}+d_{2}^{16 Q A M}}{d_{1}^{s Q P S K}+d_{2}^{s Q P S K}}=1$, i.e., the value of the modulo base for the scaled QPSK is 


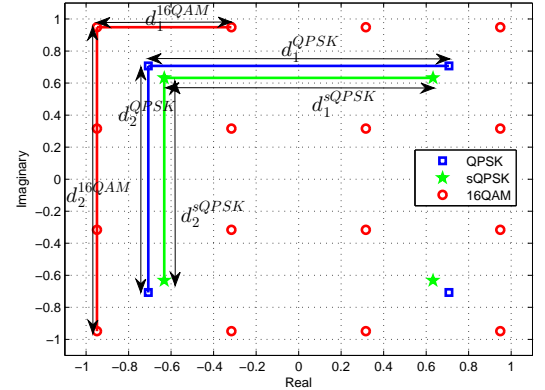

Fig. 1. Normalized constellation points for QPSK, scaled QPSK and 16QAM

equal to that of 16QAM. In this way, joint perturbation could be performed.

Remark: Although we consider a multi-stream MIMO system in this paper, the proposed algorithm is also applicable for single-stream MIMO systems. Moreover, it is obvious that our algorithm can be extended to the scenario where users apply more than two types of modulation. Finally, while for simplicity we have used instantaneous scaling factor above, the benefits of JVP directly apply to average scaling factor as well.

\section{A. Achievable Sum Rate}

Based on [5], we derive the achievable sum rate of the proposed algorithm and compare it with BD-VP and UG-VP, given as:

$$
\begin{gathered}
R_{J V P}=N_{r} \log \left(1+\frac{p_{x} \sum_{i=1}^{N_{r}} \varphi_{i}^{2}}{N_{r} \sigma_{z}^{2}}\right) \\
R_{B D-V P}=\sum_{k=1}^{K} n_{k} \log \left(1+\frac{p_{k} \sum_{i=1}^{n_{k}} \lambda_{k, i}^{2}}{n_{k} \sigma_{z}^{2}}\right) \\
R_{U G-V P}=\sum_{b=1}^{B} n_{b} \log \left(1+\frac{p_{b} \sum_{i=1}^{n_{b}} \psi_{b, i}^{2}}{n_{b} \sigma_{z}^{2}}\right)
\end{gathered}
$$

where $p_{k}=E\left\{\left\|\mathbf{x}_{k}\right\|^{2}\right\}, p_{b}=E\left\{\left\|\mathbf{x}_{b}\right\|^{2}\right\}, \mathbf{x}_{b}=\mathbf{s}_{b}+\tau_{b} \mathbf{l}_{b}, p_{x}=$ $E\left\{\|\mathbf{x}\|^{2}\right\}$, and $\mathbf{x}=\left[\left(\mathbf{s}_{\mathcal{A}}+\tau_{\mathcal{A}} \mathbf{l}_{\mathcal{A}}\right)^{T}, \ldots,\left(\mathbf{s}_{\mathcal{M}}+\tau_{\mathcal{M}} \mathbf{l}_{\mathcal{M}}\right)^{T}\right]^{T}$. $\left\{\varphi_{i}\right\}_{i=1}^{N_{r}}$ are the singular values of the equivalent channel matrix $\mathbf{H Q}$, and $\mathbf{Q}$ is obtained through $\mathrm{QR}$ decomposition of $\mathbf{H}^{\dagger} .\left\{\lambda_{k, i}\right\}_{i=1}^{n_{k}}$ are the singular values of the channel matrix $\mathbf{H}_{k} \mathbf{B}_{k} .\left\{\psi_{b, i}\right\}_{i=1}^{n_{b}}$ are the singular values of $\mathbf{H}_{b} \mathbf{Q}_{b}$, and $\mathbf{Q}_{b}$ is the null space of other group's channel matrix and can be obtained via QR decomposition.

\section{B. Complexity Analysis}

We compute the complexity in terms of the required floating point operation (flops) [10]-[11]. The inversion of the $n_{k} \times n_{k}$ matrix $\mathbf{D}_{k}$ and $N_{r} \times N_{t}$ matrix $\mathbf{H}$ using Gauss-Jordan elimination requires $4 n_{k}^{3} / 3$ flops and $4 N_{t}^{3} / 3$ flops, respectively. According to [11], the search for the optimal perturbation vector within $n$ dimensions requires $O\left(n^{6}\right)$ operations. The operator $O(\cdot)$ defines the order of numerical operators and is used to analyze the efficiency of an algorithm, also known as time complexity.
It is easy to observe that compared with the conventional VP precoding initially proposed in [4], our proposed algorithm involves the calculation of $\mathbf{G}$ and $\mathbf{t}$ which has a negligible contribution to the total complexity, therefore the complexity of the proposed algorithm is almost the same as the conventional VP algorithm. The number of flops of each algorithm needed is given as

$$
\begin{gathered}
C_{B D-V P}=4 N_{t}^{3} / 3+\sum_{k=1}^{K} O\left(n_{k}^{6}\right) \\
C_{U G-V P}=4 N_{t}^{3} / 3+\sum_{b=1}^{B} O\left(n_{b}^{6}\right) \\
C_{J V P}=4 N_{t}^{3} / 3+O\left(N_{t}^{6}\right)+2 N_{t}
\end{gathered}
$$

where it can be seen that the complexity of JVP is of the order of the conventional VP complexity.

\section{Energy Efficiency Analysis}

Energy efficiency is another metric to evaluate the usefulness of each of the VP algorithms towards an energy efficient communication system. In this section, the energy efficiency of proposed JVP is compared with previous VP algorithms in terms of the number of transmit antennas. We define the energy efficiency of the communication link as the bit rate per total transmit power consumed [8][12], shown as

$$
\varepsilon=\frac{R}{P_{P A}+N_{t} \cdot P_{0}+p_{c} \cdot C}
$$

where $P_{P A}=(\xi / \eta-1) P$ in Watts is the power consumed at the power amplifier to produce the transmit power $P$, with $\eta$ being the power amplifier efficiency and for $M$-QAM, $\xi=3 \frac{\sqrt{M}-1}{\sqrt{M}+1}$ being the modulation dependent peak to average power ratio (PAPR). $P_{0}=P_{m i x}+P_{f i l t}+P_{D A C}$ denotes the power consumption of the mixers and filters and the digitalto-analog converter (DAC), which is regarded as constant in this paper. From [13], the values of each parameter are as follows: $\eta=0.35, P_{\text {mix }}=30.3 \mathrm{~mW}, P_{\text {filt }}=2.5 \mathrm{~mW}$ and $P_{D A C}=1.6 \mathrm{~mW}$ which yields $P_{0}=34.4 \mathrm{~mW} . p_{c}$ in $\mathrm{mW} /$ Mflops denotes the power consumption per $10^{6}$ flops of the digital signal processor (DSP). In this paper we use $p_{c}=1 / 12.8 \mathrm{~mW} /$ Mflops.

\section{Numerical Results}

In this section we compare the performance of JVP, BDVP and UG-VP in adaptive modulation scenarios by means of Monte Carlo simulations. Unless stated otherwise, we assume $P=1$, the number of transmit antennas is $N_{t}=12$. There are $K=6$ users and each is equipped with $n_{k}=2$ antennas. Except for the conventional VP where all users apply QPSK, 8PSK or 16QAM, it is assumed that user 1,2 apply QPSK, user 3,4 apply 8PSK and user 5,6 apply 16QAM in BD-VP, UG-VP and JVP. Above parameters and assumptions remain the same for the following simulations. It is intuitive that the benefits of JVP extend to other adaptive modulation scenarios.

Fig. 2 shows the bit error rate (BER) performance under the adaptive modulation scenario where QPSK, 8PSK and 16QAM are applied by different users. It is obvious that JVP 


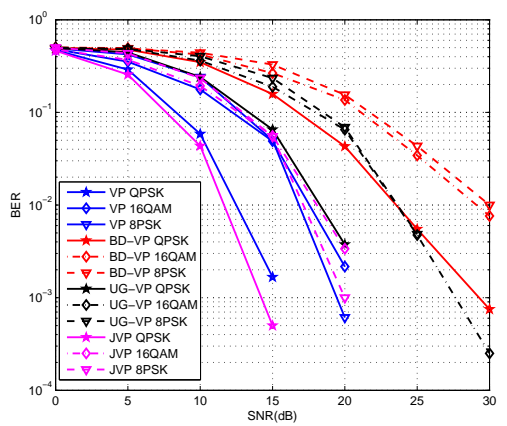

Fig. 2. Bit error rate of all users for $K=6$, transmit antenna $N_{t}=12$

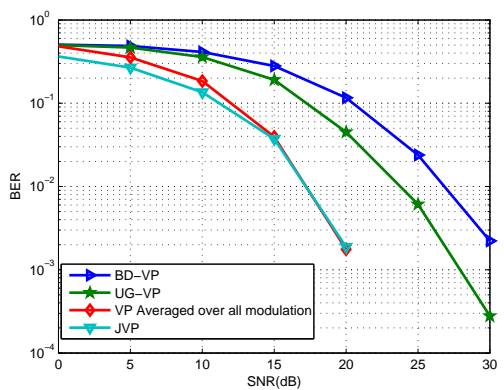

Fig. 3. Averaged bit error rate of all users for $K=6$, transmit antenna $N_{t}=$ 12

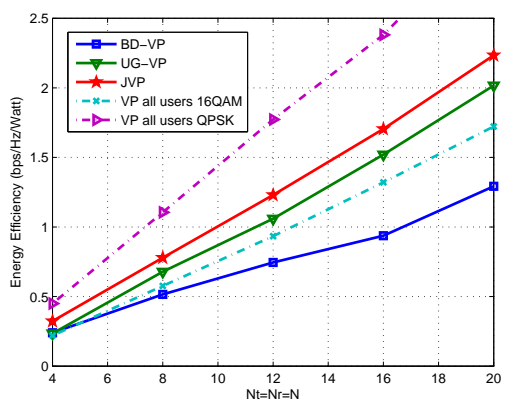

Fig. 4. Energy efficiency with the increasing number of antennas

outperforms BD-VP and UG-VP. It can also be seen that the BER performance of the users applying QPSK with the proposed algorithm is even better than the conventional VP due to the fact that the signal norm for JVP is smaller than that of QPSK. For the users applying 8PSK and 16QAM, the performance of the proposed algorithm is comparable with conventional VP.

Fig. 3 shows the BER performance averaged over all users of conventional VP, BD-VP, UG-VP and proposed joint perturbation. In this figure, 'VP Averaged over all modulation' means the averaged BER performance of VPs in which all users apply QPSK, 8PSK or 16QAM. From the figure, we can also see a similar trend as Fig. 2 that the proposed algorithm performs much better than BD-VP and UG-VP, and has a comparable performance with conventional VP algorithm.

Fig. 4 compares the energy efficiency of different VP algorithms and here we assume $P=43 \mathrm{dBm}$. From this figure, we could observe that the energy efficiency of all the VP algorithms increases with the increasing number of antennas and we could also observe that the proposed JVP outperforms BD-VP and UG-VP due to the higher achievable sum rate. Moreover, we also find that $P_{P A}$ dominates the total power consumption for all VP algorithms. VP with all users applying QPSK reaches the highest energy efficiency because the value of $\xi$ for QPSK is smaller than that of 16QAM, which makes the power consumption of VP with QPSK is smaller than that with 16QAM.

\section{CONCLUSION}

In this letter, we propose a joint vector perturbation precoding algorithm for multiuser multistream MIMO downlink system that enables the concept of VP applicable to the case where users apply different modulation types. By scaling the constellation of the modulation, the search for the perturbation vector can be performed jointly as the conventional VP so that there will be no performance loss. Simulation results validate our algorithm and show that the performance of the proposed algorithm greatly exceeds existing solutions and is comparable with the performance of the conventional vector perturbation. The combination of the proposed JVP and flexible power allocation in adaptive modulation scenarios will be a future research focus.

\section{REFERENCES}

[1] M. Costa, "Writing on dirty paper," IEEE Trans. Inf. Theory, vol. IT-29, no. 3, pp. 439-441, May 1983.

[2] L.-U. Choi and R. D. Murch, "A transmit preprocessing technique for multiuser MIMO systems using a decomposition approach," IEEE Trans. Wireless Commun., vol. 3, no. 1, pp. 20-24, Jan 2004.

[3] C. B. Peel, B. M. Mochwald, and A. L. Swindlehurst, "A vector-perturbation technique for near-capacity multiantenna multiuser communication-part I: channel inversion and regularization," IEEE Trans. Commun., vol. 53, no. 1, pp. 195-202, Jan 2005.

[4] C. B. Peel, B. M. Mochwald, and A. L. Swindlehurst, "A vector-perturbation technique for near-capacity multiantenna multiuser communication-part II: perturbation," IEEE Trans. Сотmu., vol. 53, no. 3, pp. 537-544, Mar 2005

[5] C.-B. Chae, S. Shim, and R. W. Heath, "Block diagonalized vector perturbation for multiuse MIMO systems," IEEE Trans. Wireless Commun. vol. 7, no. 11, pp. 4051-4057, Nov 2008.

[6] R. Chen. C. Li, J. Li and Y. Zhang, ”Low Complexity User Grouping Vector Perturbation," IEEE Wireless Communications Letters, vol. 1, no. 3, pp. 189-192, June 2012

[7] H. S. Han, S. H. Park, S. Lee, and I. Lee, "A decoupling approach for low-complexity vector perturbation in multiuser downlink systems," IEEE Trans. Wireless Commun., vol. 10, no. 6, pp. 1697-1701, June 2011.

[8] C. Masouros, M. Sellathurai, and T. Ratnarajah, "Maximizing Energy Efficiency in the Vector Precoded MU-MISO Downlink by Selective Perturbation," IEEE Trans. Wireless Commun., vol. 13, no. 9, pp. 4974 4984, September 2014.

[9] C. Masouros, M. Sellathurai, and T. Ratnarajah, "A Low-Complexity Sequential Encoder for Threshold Vector Perturbation," IEEE Cоттиnications Letters, vol. 17, no. 12, pp. 2225-2228, December 2013.

[10] G. H. Golub and C. F. V. Loan, Matrix Computations, 3rd edition. The Johns Hopkins University Press, 1989.

[11] H. Sung, S. CR. Lee, and I. Lee, "Generalized channel inversion methods for multiuser MIMO systems," IEEE Trans. Commun., vol. 57, no. 11, pp. 3489-3499, Nov 2009.

[12] C. Masouros, M. Sellathurai, and T. Ratnarajah, "Computationally Efficient Vector Perturbation Precoding Using Thresholded Optimization," IEEE Trans. Commun., vol. 61, no. 5, pp. 1880-1890, May 2013.

[13] C. Masouros, M. Sellathurai, and T. Ratnarajah, "Vector Perturbation Based on Symbol Scaling for Limited Feedback MISO Downlinks," IEEE. Trans. Signal Processing., vol. 62, no. 3, pp. 562-571, Feb 2014. 